\title{
OV 632 and MOC 31 in the diagnosis of mesothelioma and adenocarcinoma: an assessment of their use in formalin fixed and paraffin wax embedded material
}

\author{
C Edwards, J Oates
}

\begin{abstract}
Aims-To investigate the reaction of antibodies OV 632 and MOC 31 in paraffin wax sections as opposed to frozen sections and cytological preparations; to evaluate their usefulness in the differential diagnosis of malignant mesothelioma and secondary adenocarcinoma of the pleura; and to assess the efficacy of microwave pretreatment of sections in unmasking their associated epitopes.

Methods-Immunohistochemistry, using a standard avidin-biotin technique, with microwave pretreatment and trypsinisation in a certain proportion of cases. The material comprised 43 mesotheliomas, 44 adenocarcinomas and five reactive pleuras.
\end{abstract}

Results-Epithelial mesotheliomas and the hyperplastic mesothelial cells reacted strongly with OV 632, the reaction with sarcomatoid and desmoplastic tumours was weak, and the reaction with adenocarcinomas was variable. An unequivocal but sometimes patchy positive reaction was obtained with MOC 31 in all but one of the adenocarcinomas; all but one of the mesotheliomas and all the reactive pleuras were negative. Review of the two apparently anomalous cases revealed that the original diagnoses had probably been incorrect. Reactions to both antibodies were abolished by microwave pretreatment, and also by prior trypsinisation in the case of OV 632 .

Conclusions-OV 632 is unsuitable for routine clinical use in paraffin wax embedded material. MOC 31, however, would be a useful addition to a panel of antibodies in the differential diagnosis of mesothelioma and adenocarcinoma in large biopsy and resection specimens and biopsy specimens remains to be assessed. Microwave pretreatment does not enhance the reactions with either antibody. (f Clin Pathol 1995;48:626-630)

Histopathology, Birmingham

Heartlands Hospital, Bordesley Green East, Birmingham B9 5SS C Edwards J Oates

Correspondence to: Dr C Edwards.

Accepted for publication 3 November 1994

Keywords: OV 632, MOC 31, adenocarcinoma, mesothelioma.

The differentiation between adenocarcinomatous infiltration of the pleura and mesothelioma is often difficult, particularly in small necropsy material. Its value in small

biopsy specimens. Conventional stains for epithelial and connective tissue mucosubstances can be helpful, but lack specificity, ${ }^{12}$ and although long microvilli are almost pathognomonic of mesothelioma, material for electron microscopy is frequently unavailable. For some years now, immunocytochemical methods have been employed, using a variety of antibodies. ${ }^{1-7}$ Unfortunately, no single antibody is wholly specific: vimentin and keratins are expressed by both carcinomas and mesotheliomas; LEU M1 is not expressed by mesothelioma, but a significant proportion of adenocarcinomas are also negative; and although mesothelioma is usually negative for carcinoembryonic antigen (CEA), non-specific cross reactions frequently occur. ${ }^{356}$ Because of the lack of specificity of both immunocytochemical and conventional histochemical reactions, it is now common practice to use a battery of tests. ${ }^{35-7}$

In a recent study, ${ }^{5}$ the best combination of two markers was found to be CEA and B72.3 (both positive; $100 \%$ specific and $88 \%$ sensitive for adenocarcinoma; both negative: $99 \%$ specific and $97 \%$ sensitive for mesothelioma). These results are encouraging, but the differential diagnosis of carcinoma and mesothelioma still relies on non-specific reagents and negative results, and the search for even more reliable markers continues. With this in mind, two recently described markers-OV 632 and MOC 31 -are of interest. ${ }^{8-11}$ OV 632 appears to be particularly promising as it is a positive marker, expressed by over $90 \%$ of mesothelioma cells in cytological preparations. $^{89}$

Very little data on the behaviour of these antibodies are available, other than in cytological preparations or frozen sections. ${ }^{8-11}$ The purpose of this study was to investigate the behaviour of OV 632 and MOC 31 in formalin fixed and paraffin wax embedded material, and to assess their value in the histological diagnosis of pleural disease.

\section{Methods}

OV 632

Material from 28 cases of pleural disease was used in this part of the study, comprising 18 mesotheliomas (seven epithelial, six mixed, two sarcomatoid, and three desmoplastic), five peripheral adenocarcinomas, and five pleurectomy specimens from patients with recurrent spon- 


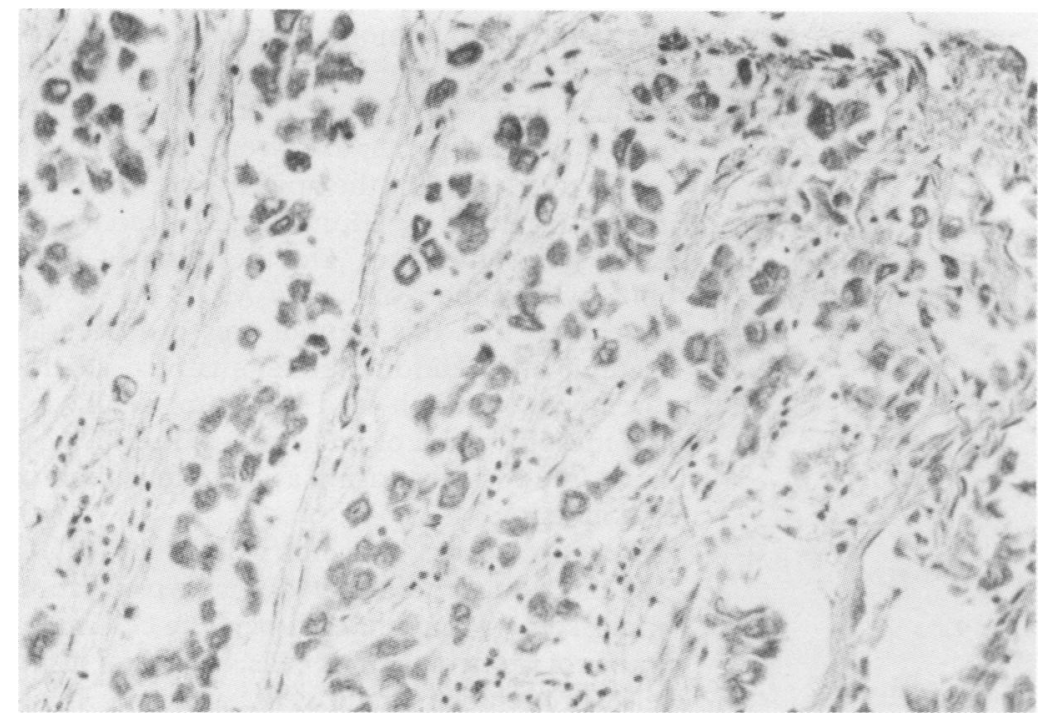

Figure 1 Immunoreactivity for OV 632 in an epithelial mesothelioma.

Reactions of the subtypes of mesothelioma, adenocarcinoma and reactive pleura with $O V$ 632

\begin{tabular}{llllll}
\hline & \multicolumn{5}{l}{ Staining intensity } \\
\cline { 3 - 6 } Tumour type & Number & Strong & Moderate & Weak & None \\
\hline Epithelial mesothelioma & 7 & 6 & 0 & 0 & 1 \\
Mixed mesothelioma & 6 & 2 & 2 & 1 & 1 \\
Sarcomatoid mesothelioma & 2 & 0 & 2 & 0 & 0 \\
Desmoplastic mesothelioma & 3 & 0 & 0 & 3 & 0 \\
Adenocarcinoma & 5 & 0 & 2 & 2 & 1 \\
Reactive pleura & 5 & 0 & 5 & 0 & 0 \\
\hline
\end{tabular}

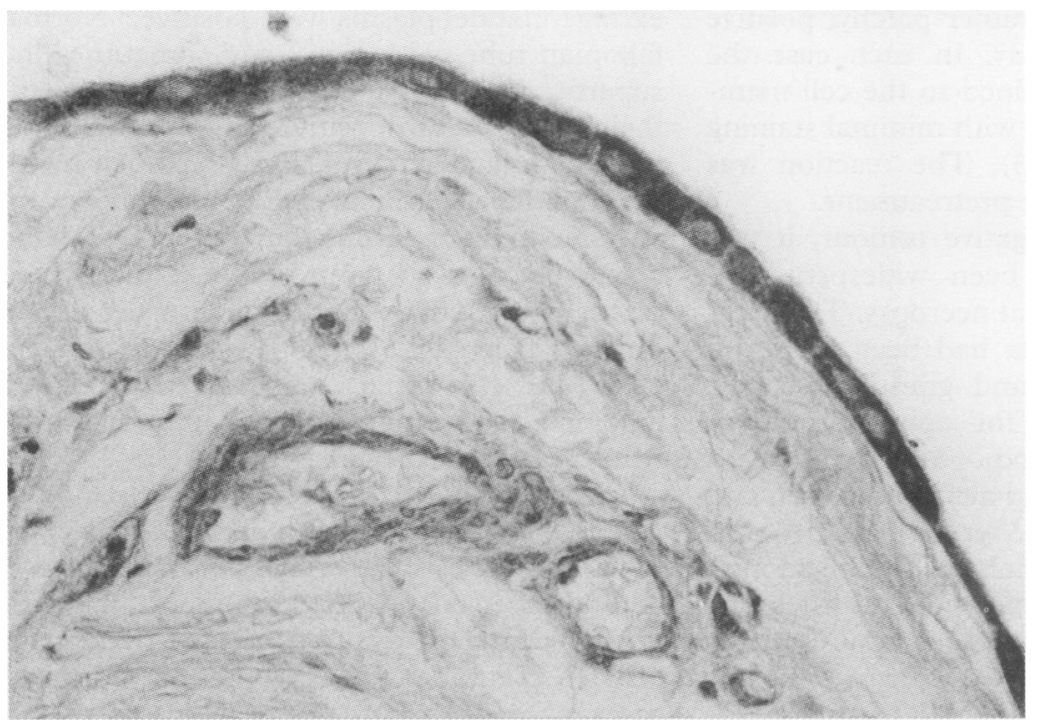

Figure 2 Immunoreactivity for OV 632 in reactive pleura from a patient with pneumothorax. immunohistochemically for OV 632 by a standard avidin-biotin complex technique, using a mouse anti-OV 632 antibody at a dilution of 1 in 10, as recommended by the manufacturers (Euro-Path Ltd, Bude, Cornwall, UK). The second section was placed in a Toshiba ER$7700 \mathrm{E}$ domestic microwave oven $(2450 \mathrm{mHz}$ : $240 \mathrm{~V}: 5 \cdot 6 \mathrm{~A}$ ) and treated at the "full power" setting for 10 minutes before staining; the third section was pretreated with trypsin by a standard method. In three of the mesothelioma cases additional sections were cut and microwave treatment was continued for 20 and $30 \mathrm{~min}$ utes. The reactions were controlled by a series of blank sections, in which the antibody was replaced by phosphate buffered saline.

\section{MOC 31}

In this part of the study a total of 87 cases was investigated. These comprised 43 mesotheliomas, of which 23 were resection specimens or large open biopsy specimens; the remainder were necropsy specimens. Twenty eight were of the epithelial subtype, eight were mixed, four were sarcomatoid, and three desmoplastic. Forty four adenocarcinomas, all of which were resection specimens or large open biopsy specimens, were also examined: 31 were of peripheral type and four were of central origin, arising in large bronchi; in five instances the exact site of origin was uncertain. Four of the tumours were metastatic deposits, all of which were from a colonic primary site. As before, specimens from five patients undergoing pleurectomy for recurrent or persistent pneumothorax were also studied.

In each case the material had been fixed in formol saline for at least 24 hours, and then processed to paraffin wax by a conventional technique. Sections were cut at $3 \mu \mathrm{m}$ and stained immunochemically for MOC 31 (EuroPath UK Ltd), using an avidin-biotin technique and an antibody dilution of 1 in 20 . At the beginning of this part of the investigation, sections from five of the adenocarcinomas and five of the mesotheliomas were pretreated in a domestic microwave oven as above for 10 minutes.

\section{Results}

OV 632

The reactions of the mesotheliomas, the adenocarcinomas and the reactive pleuras, together with the effects of trypsinisation and microwave pretreatment are shown in the table. The strongest reaction was seen in the epithelial mesotheliomas and in the epithelial component of the mixed mesotheliomas (fig 1). Desmoplastic mesotheliomas gave only a weakly positive reaction, whereas sarcomatoid tumours occupied an intermediate position. The reaction in three of the adenocarcinomas was weak or equivocal, strong in one and negative in another. The five reactive pleurae showed clear positive staining (fig 2). Contrary to expectation, microwave pretreatment abolished or noticeably reduced the strength of the reaction and when sections were trypsinised, there was no reaction whatsoever. 


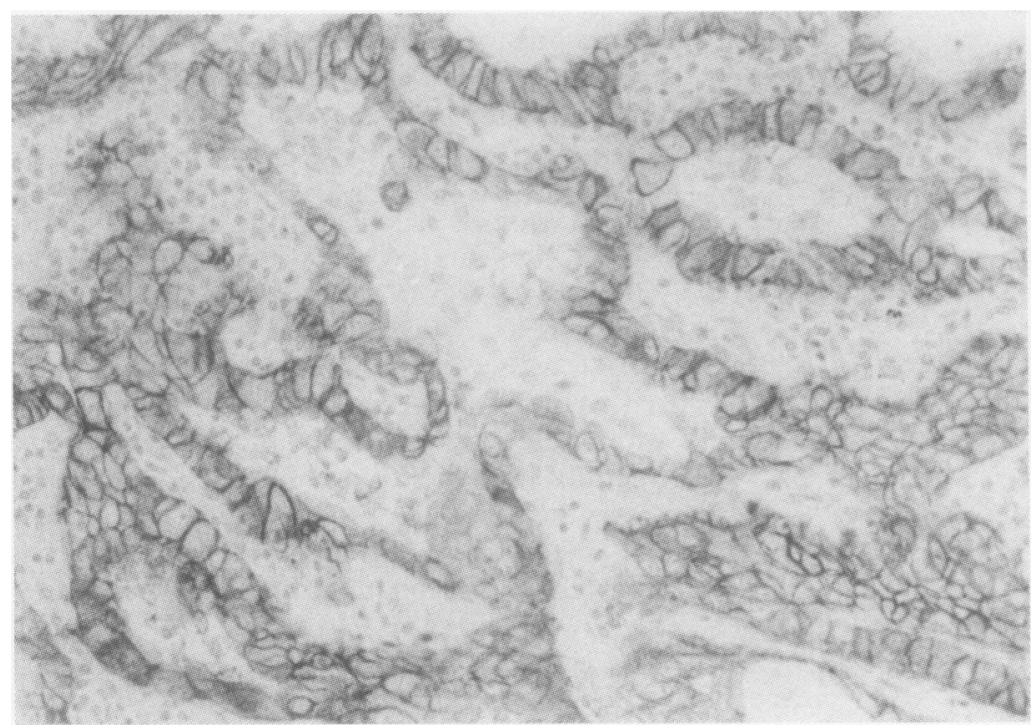

Figure 3 Immunoreactivity for MOC 31 in a pulmonary adenocarcinoma.

There was no consistent reaction in the lung parenchyma adjacent to the adenocarcinomas, but the overlying normal pleura gave a positive reaction which was abolished by both microwave pretreatment and trypsinisation. In all of the sections examined microwave pretreatment induced a positive cytoplasmic reaction in alveolar and non-alveolar macrophages.

\section{MOC 31}

All but one of the adenocarcinomas gave a strong, but sometimes rather patchy, positive reaction to this antibody. In each case the reaction was either confined to the cell membrane, or was associated with minimal staining of the cytoplasm (fig 3). The reaction was quenched by microwave pretreatment.

On review of the negative tumour, it was found that there had been widespread involvement of the pleura at necropsy. The diagnosis of adenocarcinoma had been made on the basis of a scanty and granular positive histochemical reaction for epithelial mucosubstances, using periodic acid-Schiff with and without diastase, and alcian blue with and without hyaluronidase. Morphologically, with haematoxylin and eosin alone, the distinction between adenocarcinoma and mesothelioma was not possible and the exact nature of this tumour remains uncertain.

With a single exception, all of the mesotheliomas were negative, although in some cases there were small, probably artefactual foci of positivity, involving isolated cells or small clusters. There was no unmasking of the epitope by microwave pretreatment or trypsinisation. The positive tumour showed the same heavy membrane reaction seen in the adenocarcinomas. Pleural infiltration characteristic of mesothelioma had been found at necropsy, but there had been no history of asbestos exposure and no asbestos was detected in the lung parenchyma by either light or electron microscopy. The patient had, however, been treated for Hodgkin's lymphoma at six years of age. A careful review of the sections revealed that the tumour consisted for the most part of regular acini lying in a bland fibrous stroma, lined by cuboidal or low columnar cells with open nuclei; the cytoplasm was often vacuolated. Overall, the appearance was more in keeping with a peripheral adenocarcinoma than a mesothelioma. On the basis of morphology and the reaction to MOC 31, it was concluded that this tumour was probably an example of "pseudomesotheliomatous" adenocarcinoma, ${ }^{12}$ which had been misdiagnosed as a mesothelioma of uncertain (non-asbestos) aetiology, possibly arising as a complication of radiotherapy. ${ }^{13}$

Reactive pleura was not stained by MOC 31 , and neither microwave nor trypsinisation unmasked any hidden epitope. The cell membranes of bronchial lining cells, bronchial gland cells, bronchiolar lining cells, and type II alveolar lining cells were positive; type I cells appeared as a thin line of reaction product coating the internal surface of the alveoli. The reaction was abolished by microwave pretreatment and by trypsinisation. Normal pleura was negative.

\section{Discussion}

OV 632 is a hybridoma cell line developed from the spleen cells of a mouse immunised with fluid from a serous cystadenoma. ${ }^{8}$ It was found that the monoclonal antibody produced by these cells reacted with 22 of 28 non-mucinous carcinomas and three of seven mucinous carcinomas of the ovary; only seven of 122 extragenital neoplasms were positive. ${ }^{8}$ Normal fallopian tube epithelium, and sometimes the superficial edometrium and endocervical epithelium, were also positive, as were the lining cells of follicular cysts. On the other hand, the superficial germinal epithelium of the ovary and the lining of germinal inclusion cysts were negative. The exact nature of the epitope which reacts with OV 632 is uncertain, but its reaction in the female genital tract suggests that it is an early Mullerian antigen which is retained in the fallopian tube and reappears in tumours of Mullerian-derived tissues. ${ }^{8}$

In embryological terms ovarian carcinomas and malignant pleural tumours share a common histogenesis from coelomic mesoderm. ${ }^{9}$ This theoretical relation prompted an evaluation of the reactivity of OV 632 with malignant cells in pleural fluids. Preparations from 43 cases of mesothelioma and 62 cases of adenocarcinoma were examined and over $90 \%$ of the mesothelioma specimens gave a positive reaction, compared with only two of the 22 cases of non-ovarian carcinoma. ${ }^{9}$

In the original study of OV $632^{8}$ only frozen sections were used because it was found that there was no reaction with formalin fixed and paraffin wax embedded material. Even in frozen sections the reaction was blocked by a short incubation in $2 \%$ formalin. A single mesothelioma examined at this time was reported to be negative.

Despite these obvious shortcomings, it was clear that OV 632 required further study. The antibodies in routine use rely on a negative 
reaction for mesothelioma and a positive reaction for carcinoma, an unsatisfactory state of affairs which may lead to diagnostic errors. ${ }^{3-7}$ OV 632 , on the other hand, appeared to be unique in that it gives a positive reaction with mesothelioma, albeit in cytological preparations. If the blocking of the reaction by routine fixation and processing could be overcome, it would be a valuable addition to the battery of antibodies currently used in this context. ${ }^{3-7}$ It has recently been shown that microwave irradiation often enhances immunohistochemical reactions, and may "retrieve" epitopes that have previously only been demonstrable in frozen sections. ${ }^{1415}$ Accordingly, we investigated the effect of microwave pretreatment on the reactivity of OV 632 with paraffin wax embedded and formalin fixed material, in the hope that the epitope would be unmasked.

The results of this part of the study were unexpected in that the antibody reacted strongly with untreated sections of most of the epithelial mesotheliomas, and rather less strongly with the sarcomatoid and desmoplastic mesotheliomas. Untreated sections of reactive pleura were also strongly positive and the reaction with adenocarcinomas was variable. Both the microwaved and trypsinised sections were negative. These results are at variance with those obtained by other investigators ${ }^{8-10}$; we have no explanation except that they may be due to the use of a different batch of antibody. It is certain, however, that although the antibody we used reacted in a way that merits further study, OV 632 cannot be relied upon to differentiate between adenocarcinoma and mesothelioma in paraffin wax sections.

MOC 31 is the prototype reagent of cluster 2 as defined at the Second International Workshop on Lung Cancer Antigens. ${ }^{116}$ It recognises a $40 \mathrm{kDa}$ transmembrane glycoprotein of uncertain function, located on the membrane of certain epithelial cells, including those of adenocarcinoma. To our knowledge, MOC 31 has only been evaluated in two studies, both of which were cytological. In the first, ${ }^{9}$ MOC 31 positivity was seen in cells from effusions in seven of 10 adenocarcinomas of lung, and in 11 of 20 specimens from patients with effusions due to adenocarcinomas from other sites: only one of seven effusions arising as a result of secondary carcinoma of the breast contained positive cells. Fluid samples from 24 mesotheliomas were examined, and only two were positive. These data were re-presented by the same group in a further paper two years later. ${ }^{10}$

In the second investigation, ${ }^{11}$ in which effusions from 98 patients were studied, positive cells were seen in all but one of 59 cases of secondary carcinoma. The primary sites included lung, breast and thyroid, and the gastrointestinal and genito-urinary tracts. The single exception was a case of secondary squamous carcinoma. The cells in the fluid samples from 29 patients with reactive effusions, and from five with mesothelioma, were negative. The authors concluded that MOC 31 is a highly selective and reliable discriminator between epithelial and mesothelial cells, and is certainly more specific and sensitive than the other antibodies used in the study, which included CEA, epithelial membrane antigen, cytokeratins of differing molecular weights, and vimentin.

Our experience with this antibody indicates that it is equally effective in formalin fixed, paraffin wax embedded material, and that in discriminating between adenocarcinomatous infiltration of the pleura and malignant mesothelioma, it compares favourably in its performance with other antibodies. Leu M1, for instance, reacts with about $75 \%$ of adenocarcinomas and with up to $6 \%$ of mesotheliomas ${ }^{35}$; B72.3 has recently been reported to react with 93 of 103 adenocarcinomas, whereas 34 mesotheliomas were negative. ${ }^{5}$ In this same study CEA reacted with 100 of the adenocarcinomas and with only three of the mesotheliomas: less specific results were obtained with secretory component and CA 125, while vimentin and thrombomodulin were unhelpful.

The lack of specificity of the various antibodies that have been advocated from time to time to assist the differential diagnosis of these two tumours and the absence of a positive antibody for mesothelioma have led to the use of panels of antibodies. Currently, the most useful panel would appear to consist of CEA, Leu $M 1$ and B72 $3,{ }^{5}$ but even then some care must be taken in the selection of reagents. There are, for instance, numerous antibodies to CEA which recognise at least five epitope clusters, each with different sensitivities and specificities, ${ }^{6}$ and some published data suggest that B72.3 may react with $21 \%$ of mesotheliomas. ${ }^{5}$ Moreover, the results of any investigation are, as Weiss and Battifora ${ }^{6}$ so rightly point out, "only as good as the original pathologic diagnosis". The purpose of a panel of immunochemical tests is to aid diagnosis and cannot be regarded as the solution to all problems.

The results of our study indicate that MOC 31 is potentially a most useful addition to a panel of antibodies for the diagnosis of pleural malignancy. It must be stressed, however, that so far this antibody has only been evaluated in the context of large open biopsy specimens, reaction specimens, and necropsy material. Its value in small biopsy specimens remains to be elucidated; the patchy nature of the staining in some adenocarcinomas and the small foci of positivity in some mesotheliomas may prove troublesome in practice. Unfortunately, despite its original promise, OV 632 cannot be recommended for use in formalin fixed, paraffin wax embedded material. Neither antibody is enhanced by either microwave pretreatment or prior trypsinisation of the sections.

We are grateful to Euro-Path Ltd for providing the antibodies used in this investigation, which was also supported in part by Celltech UK Ltd.

1 Roggli VL, Kolbeck J, Sanfilipo F, Shelburne JD. Pathology of human mesothelioma: etiologic and diagnostic considerations. Pathol Annu 1987;22:91-133.

2 Henderson DW, Shilkin KB, Whitaker D, Attwood HD, Constance TJ, Steele RH, et al. The pathology of malignant mesothelioma, including immunohistology and ultrastructure. In: Henderson DW, Shilkin KB, Langlois 
SL, Whitaker D, eds. Malignant mesothelioma. New York: Hemisphere Publishing Corporation, 1992:69-139.

3 Sheibani K, Esteban JW, Bailey A, Battifora H, Weiss LM. Immunopathologic and molecular studies as an aid to the diagnosis of malignant mesothelioma. Hum Pathol 1992; 23:107-16.

4 Frisman DM, McCarthy WF, Schleiff P, Buckner S-B, Nocito D, O'Leary TJ. Immunochemistry in the differential diagnosis of effusions: use of logistic regression to ential diagnosis of effusions: use of logistic regression to
select a panel of antibodies to distinguish adenocarcinomas from mesothelial proliferations. Mod Pathol 1994;6:179 84

5 Brown RW, Clark GM, Tandon AT, Allred DC. Multiplemarker immunohistochemical phenotypes distinguishing malignant pleural mesothelioma from pulmonary adenocarcinoma. Hum Pathol 1993;24:347-54.

6 Weiss LM, Battifora $\mathrm{H}$. The search for the optimal immunohistochemical panel for the diagnosis of malignant mesothelioma. Hum Pathol 1993;24:345-6.

7 Sheibani K. Immunopathology of malignant mesothelioma Hum Pathol 1994;25:219-20.

8 Fleuren GJ, Coerkamp EG, Nap M, Van den Broek LJCM, Warnaar SO. Immunohistological characterization of monoclonal antibody (OV 632) against epithelial ovarian carcinomas. Virchows Arch A Pathol Anat 1987;410:481-6.

9 Delahaye M, Hoogsteden HC, van der Kwast TH. Immunocytochemistry of malignant mesothelioma: OV 632 as a marker of malignant mesothelioma. 7 Pathol 1991; 165:137-43.

10 van der Kwast TH, Delahaye M, Versnel M, Hoogsteden $\mathrm{H}$. Improvement of the cytological diagnosis of malignant mesothelioma. Eur Respir Rev 1993;11:57-8.

11 Ruitenbeck T, Gouw ASH, Poppema S. Immunocytology of body fluids: MOC-31, a monoclonal antibody discriminating between mesothelial and epithelial cells. Arch criminating between mesothelial and epithelial cells. Arch

12 Koss M, Travis W, Moran C, Hocholzer L. Pseudomesotheliomatous adenocarcinoma: a reappraisal. Semin Diag Pathol 1992;9:117-23.

13 Austin MB, Fechner RE, Roggli VL. Pleural malignant mesothelioma following Wilms' tumour. Am f Clin Pathol 1986;86:227-30.

14 Shi S-R, Key ME, Kaltra KL. Antigen retrieval in formalinfixed, paraffin embedded tissues: an enhancement method for immunohistochemical staining based on microwave oven heating of tissue sections. $\mathcal{F}$ Histochem Cytochem 1991; 39:741-8.

15 Cuevas EC, Bateman AC, Wilkins BS, Johnson PA, Williams $\mathrm{JH}$, Lee AHS, et al. Microwave antigen retrieval in immunocytochemistry: a study of 80 antibodies. $\mathcal{F}$ Clin Pathol munocytochemis

16 Souhami RL. The antigens of lung cancer. In: Thatcher N, Spiro S, eds. New perspectives in lung cancer. London: BMJ Publishing Group, 1994:68-76. 